\title{
Effect of Dimensionality on The Electronic Properties of SnSe: A Density Functional Theory Study
}

\author{
Erik Bhekti Yutomo, Fatimah Arofiati Noor*, Toto Winata \\ Physics of Electronics Materials Research Division, Department of Physics, Faculty of Mathematics and Natural \\ Sciences, InstitutTeknologi Bandung, Bandung 40132, Indonesia.
}

(Received: December 8, 2021, Revised: December 23, 2021, Accepted: December 29, 2021)

\begin{abstract}
This study investigated the structural and electronic properties of bulk, bilayer, and monolayer SnSe using the density functional theory (DFT) method. We succeeded in calculating the bandgap and identifying accurately the transformation of the band structure from bulk to monolayer systems using generalized gradient approximation. An increase in the lattice parameter $a$ and a decrease in the lattice parameter $b$ were observed when the bulk dimensions were reduced to a monolayer. The reduction of van der Waals interactions when the dimensions of a system are reduced is the main factor that causes changes in lattice parameters. The indirect bandgap of bulk SnSe $(0.56 \mathrm{eV}, 0.3 \Delta \rightarrow 0.7 \Sigma)$ becomes wider in the monolayer system $(0.94 \mathrm{eV}, 0.2 \Delta \rightarrow 0.8 \Sigma)$. Bandgap widening is predicted due to the emergence of the quantum confinement effect in low-dimensional systems. Furthermore, we found the formation of a quasi-degenerate minimum conduction band in the monolayer SnSe. With the formation of these bands, we predict the monolayer SnSe will have better thermoelectric properties than the bulk or bilayer system. This study provides an in-depth understanding of the electronic structure of $\mathrm{SnSe}$ and its correlation to thermoelectric properties.
\end{abstract}

Keywords: Bandgap, electronic properties, monolayer $\mathrm{SnSe}$, thermoelectric properties.

\section{INTRODUCTION}

Following the isolation of graphene for the first time, theoretical and experimental research on optimizing the physical properties of graphene has increased [1-4]. The superior mechanical, electronic, and thermal properties of graphene cannot be separated from the quantum confinement effect due to dimensional reduction [5]. However, its high thermal conductivity and zero bandgap characteristics make graphene less suitable for thermoelectric and optoelectronic device applications [6,7]. These conditions motivated researchers to look for other 2D materials with exciting properties, such as members of the layered group IVmonochalcogenides MX $(M=\mathrm{Ge}, \mathrm{Sn} ; \quad \mathrm{X}=\mathrm{S}, \mathrm{Se}$, $\mathrm{Te})$ [8-10]. Monolayer tin sulfide $(\mathrm{SnS})$ is a p-type semiconductor with a moderate bandgap of $1.1 \mathrm{eV}$ [11]. By using a charge transfer mechanism, monolayer $\mathrm{SnS}$ has potential as an acetone sensor for diabetes detection [9]. As a result of a unique electronic structure giving it a low energy difference between its two maximum valence band extremes, monolayer $\mathrm{SnS}$ has good thermoelectric properties [11]. Monolayer GeSe exhibits a direct bandgap of $1.59 \mathrm{eV}$ and high charge carrier mobility of 4032.64 $\mathrm{cm}^{2} \mathrm{~V}^{-1} \mathrm{~s}^{-1}$ [12]. Such conditions make monolayer GeSe a potential material for use in photovoltaic applications. The bandgap can be adjusted by reducing the number of layers [12]. The characteristics of Mexican-hat on the electronic structure of monolayer $\mathrm{GeSe}$ can induce ferroelectric properties with high Curie temperatures [13].

Recently, single-layer single-crystalline tin selenide $(\mathrm{SnSe})$ was successfully synthesized using the one-pot colloidal route method [14]. The morphology of the resulting monolayer $\mathrm{SnSe}$ is highly dependent on the concentration of control agents such as phenanthroline, which regulates growth kinetics [14]. Monolayer SnSe shows an indirect bandgap of $0.86 \mathrm{eV}$, which means it is in the range of the energy spectrum of sunlight [14]. Furthermore, monolayer $\mathrm{SnSe}$ exhibits excellent photoresponse properties such as high photocurrent,

\footnotetext{
${ }^{*}$ Corresponding author.

E-mail address: fatimah@fi.itb.ac.id
} 
high response time, and high on/off ratio [14]. From the computational results, monolayer $\mathrm{SnSe}$ exhibits an indirect bandgap of $0.91-1.12 \mathrm{eV}[15,16]$. However, the modified Becke-Johnson (mBJ) approximation overestimates the bandgap [17]. The inclusion of weak van der Waals interactions also results in less precise bandgap calculations [15,17]. With the application of a slight strain, the energy gap of monolayer $\mathrm{SnSe}$ undergoes an indirect-direct transformation [17]. The low thermal conductivity $\left(<3 \mathrm{Wm}^{-1} \mathrm{~K}^{-1}\right.$ at $\left.300 \mathrm{~K}\right)$ and the high charge carrier mobility $\left(10000 \mathrm{~cm}^{2} \mathrm{~V}^{-1} \mathrm{~S}^{-1}\right)$ of monolayer $\mathrm{SnSe}$ make it a potential material for thermoelectric and photovoltaic devices [14,17]. However, there are limited reports that discuss the transformation that occurs in the electronic structure when the dimensions of bulk $\mathrm{SnSe}$ are reduced to lowdimensional nanostructures such as bilayer and monolayer systems.

In this paper, we report a comparison of the structural and electronic properties of bulk, bilayer, and monolayer $\mathrm{SnSe}$ calculated using the density functional theory (DFT) method. We demonstrate a correlation between the transformation of electronic structure and the improvement of physical properties, especially thermoelectric properties. We employed generalized gradient approximation (GGA) and projector augmented wave (PAW) methods. We reveal the increase in band convergence in the monolayer $\mathrm{SnSe}$ system. Band convergence is very advantageous in improving the thermoelectric properties of the related material.

\section{COMPUTATIONAL METHOD}

In this study, bulk SnSe was modeled with its unit cell (space group: Pnma) consisting of four Sn atoms and four $\mathrm{Se}$ atoms. The initial lattice parameters were taken from the experimental results, that is, $a=4.135 \AA, b=4.440 \AA$, and $c=11.490 \AA$, and the distance between the adjacent layers $d_{12}=$ $2.712 \AA$ [18]. For the bilayer SnSe system, the AAtype stacking configuration was chosen because it has better structural stability than other types [19]. The initial lattice parameters and the distance between the adjacent layers of the bilayer SnSe system were adopted from the optimized geometric structure of the bulk SnSe system. Subsequently, a vacuum of $20 \AA$ was applied to the system to avoid interactions between nearby layers so that the system consisted of only four $\mathrm{Sn}$ atoms and four Se atoms. When modeling the monolayer SnSe system, the same treatment as for the bilayer SnSe system was also applied, so that the system consisted of two Sn atoms and two Se atoms.
All DFT calculations were performed using Quantum ESPRESSO codes [20,21]. Pseudopotential with PAW type was chosen to regulate the interaction of ion and valence electrons [22]. This type considers only $4 \mathrm{~d}, 5 \mathrm{~s}$, and $5 \mathrm{p}$ electron configurations for $\mathrm{Sn}$ atoms and $3 \mathrm{~d}, 4 \mathrm{~s}$, and $4 \mathrm{p}$ for $\mathrm{Se}$ atoms. Furthermore, the exchange-correlation interaction between valence electrons was approximated by a GGA based on the PerdewBurke-Ernzerhof (PBE) functional [23]. This function successfully predicted the electronic and magnetic properties of the low-dimensional system in our previous study $[3,4]$.

A cut-off energy of $\sim 1224 \mathrm{eV}$, a threshold energy of $\sim 13.6 \mathrm{meV}$, and a threshold force of $\sim 25$ $\mathrm{meVA}^{-1}$ were used in self-consistent field (SCF) calculations for bulk, bilayer, and monolayer $\mathrm{SnSe}$ systems. However, because the dimensions of the three systems are different, k-points of $7 \times 7 \times 3$ and $7 \times 7 \times 1$ were used for the bulk $\mathrm{SnSe}$ and the lowdimensional $\mathrm{SnSe}$ (bilayer and monolayer), respectively. Calculation of the electronic structure was carried out through the $\Gamma-\mathrm{X}-\mathrm{S}-\mathrm{Y}-\Gamma$ path in the irreducible Brillouin zone. Before calculating the electronic properties, the lattice parameters and atomic positions in each system were optimized using the Broyden-Fletcher-Goldfarb-Shanno (BFGS) method [24-27]. The DFT-D3 semiempirical correction by Grimme was included to represent weak van der Waals interactions between adjacent layers in bulk and bilayer systems during geometry optimization [28].

\section{RESULTS AND DISCUSSION}

Figure 1 shows the geometry structure of bulk, bilayer, and monolayer $\mathrm{SnSe}$, while the optimized structural parameters are summarized in Table 1. The calculated lattice parameters for the bulk system are slightly larger than the previous experimental results [18]. This condition can occur because we use the GGA-PBE functional, which tends to overestimate the lattice parameters. However, our results are consistent with previous computational studies [29].

Table 1. Lattice parameters, bond lengths, and bond angles of bulk, bilayer, and monolayer SnSe.

\begin{tabular}{ccccc}
\hline \multirow{2}{*}{$\begin{array}{c}\text { Parameter } \\
\mathrm{s}\end{array}$} & \multicolumn{2}{c}{ Bulk } & \multirow{2}{*}{ Bilaye } & Monolaye \\
\cline { 2 - 4 } & $\begin{array}{c}\text { Ref. } \\
{[18]}\end{array}$ & $\begin{array}{c}\text { This } \\
\text { study }\end{array}$ & $\mathrm{r}$ & $\mathrm{r}$ \\
\hline$a(\AA)$ & 4.14 & 4.17 & 4.26 & 4.28 \\
$b(\AA)$ & 4.44 & 4.56 & 4.47 & 4.43 \\
$c(\AA)$ & 11.4 & 11.59 & 28.88 & 23.08 \\
$l_{1}(\AA)$ & 9 & 2.79 & 2.76 & 2.73 \\
$l_{2}(\AA)$ & & 2.81 & 2.88 & 2.90
\end{tabular}




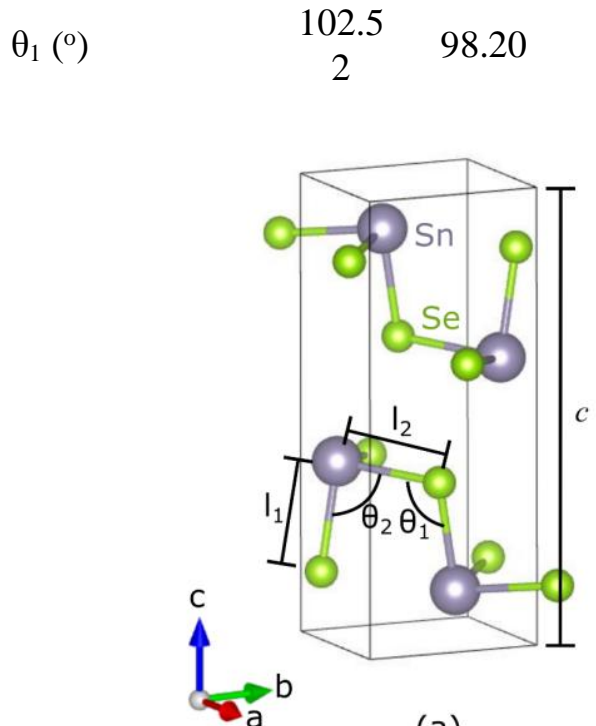

(a)

94.38

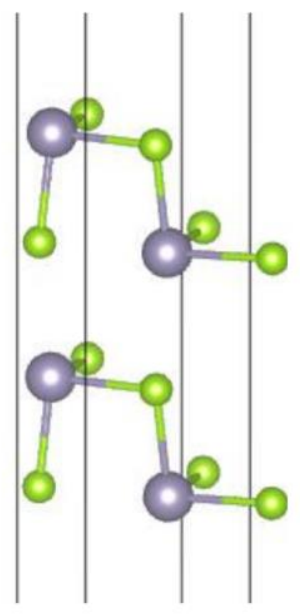

(b)

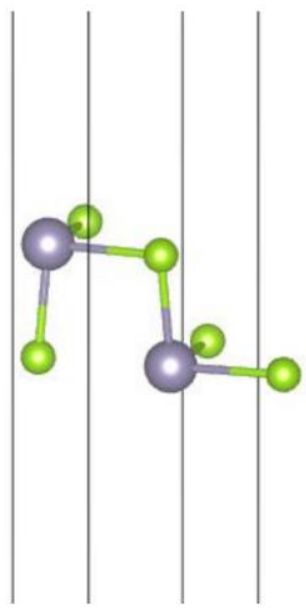

(c)

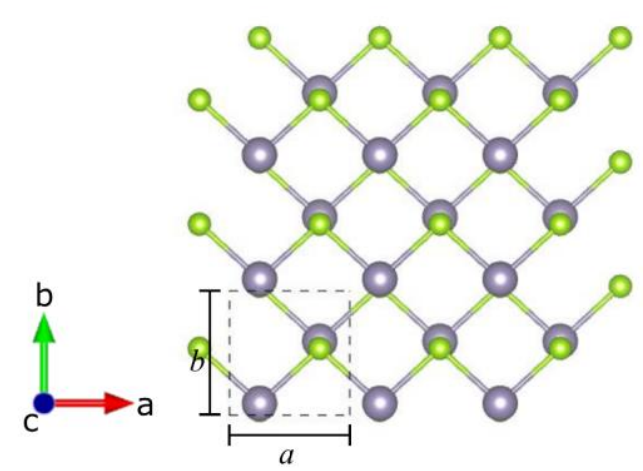

(d)

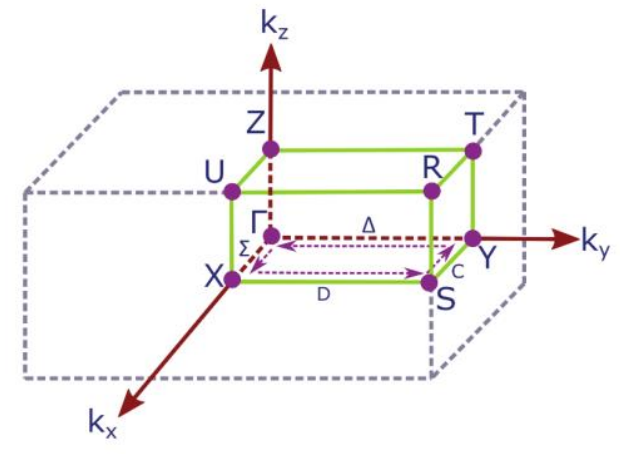

(e)

Figure 1. (a-c) side views of the geometric structures of the systems, (d) top view of the geometric structure of the SnSe monolayer system, (e) first order Brillouin zone with paths for electronic structure calculations.

The formation of nanostructures significantly changes the structural parameters of the SnSe system. From bulk to monolayer system, bond length $l_{1}$ and bond angle $\theta_{1}$ decreases while $l_{2}$ and $\theta_{2}$ increases. This condition causes an increase in lattice parameter $a$ and a decrease in lattice parameter $b$ from bulk to monolayer $\mathrm{SnSe}$ system. Changes in lattice parameters due to reduction in system dimensions are closely related to the elimination of van der Waals interactions, especially for monolayer systems. Furthermore, bilayer SnSe has a greater interlayer distance, $d_{12}$, than bulk SnSe. This condition can occur because, in bilayer $\mathrm{SnSe}$, the coordination number of the atoms in the first and second layers is reduced, so that these layers become freer to relax in the opposite direction. Changes in the geometric structure parameters are predicted to induce significant changes in the electronic structure.

Figure 2 shows the band structure of the bulk, bilayer, and monolayer SnSe system. The band structure is calculated along the $\Gamma-\mathrm{X}-\mathrm{S}-\mathrm{Y}-\Gamma$ path in the first order Brillouin zone, as illustrated by Figure 1(e). Furthermore, Table 2 summarizes the direct and indirect bandgaps of all systems. As a result, bulk, bilayer, and monolayer SnSe systems have almost the same band dispersion in both the valence and conduction bands, especially for the bilayer and monolayer systems.

Table 2. The bandgaps of bulk, bilayer, and monolayer $\mathrm{SnSe}$.

\begin{tabular}{ccc}
\hline \multirow{2}{*}{ System } & \multicolumn{2}{c}{ Bandgap $(\mathrm{eV})$} \\
\cline { 2 - 3 } Bulk & $0.56(0.3 \Delta \rightarrow 0.7 \Sigma$ & $0.85(0.6 \Sigma \rightarrow 0.6 \Sigma$ \\
& Indirect & Direct \\
Bilayer & $0.84(0.2 \Delta \rightarrow 0.9 \Sigma$ & $0.95(0.2 \Delta \rightarrow 0.2 \Delta$ \\
Monolaye & $0.94(0.2 \Delta \rightarrow 0.8 \Sigma$ & $0.99(0.2 \Delta \rightarrow 0.2 \Delta$ \\
$\mathrm{r}$ & ) & ) \\
\hline
\end{tabular}

In Figure 2(a), bulk SnSe shows an indirect bandgap of $0.56 \mathrm{eV}$. The maximum valence band lies at $0.3 \Delta$, and the minimum conduction band lies at $0.7 \Sigma$, where $\Delta$ and $\Sigma$ are paths connecting $\mathrm{Y}-\Gamma$ and $\Gamma-\mathrm{X}$ points, 
respectively. Our calculated bandgap is smaller than the experimental measurement of optical gap energy of $0.86 \mathrm{eV}$ [18]. This is due to the GGA-PBE functional, which has a limitation in calculating the bandgap. However, our calculation results are in the range of the previous computational result of 0.44 $0.89 \mathrm{eV}$ [29-33], which uses the LDA and GW functional types. We observe the presence of the multi-valley band, which is one of the exciting characteristics of the band structure of bulk SnSe because it can be exploited to increase the thermopower properties.
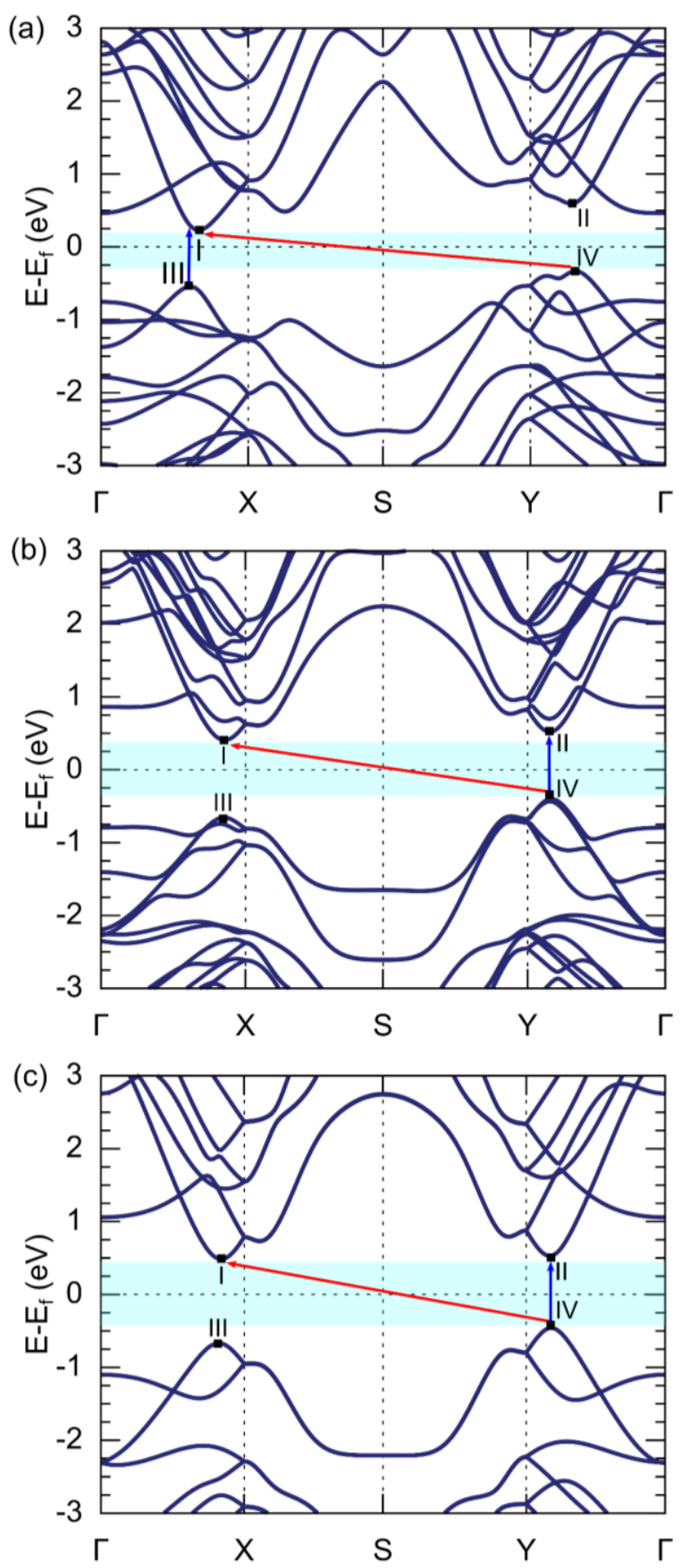

Figure 2. Band structures of (a) bulk, (b) bilayer, and (c) monolayer $\mathrm{SnSe}$. Cyan-colored areas denote bandgap, red and blue arrows indicate indirect and direct bandgaps, respectively, and numbers I-IV indicate the extreme band in the valence and conduction bands.

In Figure 2(b), the bilayer $\mathrm{SnSe}$ shows an indirect bandgap with a magnitude of $0.84 \mathrm{eV}(0.2 \Delta \rightarrow 0.9 \Sigma)$. This value was obtained using the functional GGAPBE. Our results are consistent with the previously computed result of $0.88 \mathrm{eV}$ [19]. The electronic structure of bilayer $\mathrm{SnSe}$ has interesting characteristics in the valence and conduction bands, that is, the "pudding mold" band. This band consists of a flat band on the X-S-Y path, with some part of the corrugation band ending with a highly dispersive curved band on the $\Gamma-\mathrm{X}$ and $\mathrm{Y}-\Gamma$ paths $[34,35]$. However, the flat band part undergoes bending in the conduction band. Four highly dispersed bands, as marked in Figure 2(b), potentially increase the magnitude of the Seebeck coefficient through the band convergence scheme [36-38]. In this system, the extrema I and II bands at the conduction band still have a significant difference, as shown in Table 3. Notably, this value is smaller than in the bulk system.

Table 3. Energy difference between extreme bands III $\Delta E_{\text {I-II }}$ and extreme bands III-IV $\Delta E_{\text {III-IV }}$

\begin{tabular}{ccc}
\hline System & $\Delta E_{\text {I-II }}(\mathrm{eV})$ & $\Delta E_{\text {III-IV }}(\mathrm{eV})$ \\
\hline Bulk & 0.36 & 0.21 \\
Bilayer & 0.11 & 0.21 \\
Monolayer & 0.05 & 0.21 \\
\hline
\end{tabular}

In monolayer $\mathrm{SnSe}$, the bandgap increases to $0.94 \mathrm{eV}$ due to quantum confinement. The bandgap remains indirect; the position of the maximum conduction band shifts only slightly due to lattice distortion. Our result is greater than the previous experimental result of $0.86 \mathrm{eV}$ [14]. However, our results are consistent with previous computational studies [16]. By incorporating a semiempirical van der Waals correction, the bandgap of the monolayer SnSe increases to $1.12 \mathrm{eV}[15,17]$. This condition indicates that the weak van der Waals bond does not exist in the monolayer SnSe system. Moreover, the accuracy reduces if allowance is made for a weak van der Waals bond in the calculation. An overestimation of the bandgap also resulted when using the modified Becke-Johnson (mBJ) method [17]. Thus, the functional GGA-PBE is more suitable for estimating the bandgap of the monolayer $\mathrm{SnSe}$ system. An essential aspect of the electronic structure of the SnSe monolayer is the appearance of a quasi-degenerate maximum conduction band. Consequently, the energy difference of the extreme I and II bands is reduced, as shown in Table 3. This condition is predicted to induce better thermoelectric properties for the monolayer system than the bulk and bilayer 
systems, especially from the Seebeck coefficient aspect $[38,39]$. However, it is necessary to evaluate the thermoelectric properties of bulk, bilayer, and monolayer $\mathrm{SnSe}$ systems to prove our predictions. Therefore, it will be our future study and will be published elsewhere.

\section{CONCLUSION}

We revealed the transformation of the structural and electronic properties of bulk, bilayer, and monolayer SnSe. The formation of nanostructures (monolayer and bilayer) makes the lattice parameter, $a$, increase and the lattice parameter, $b$, decrease. The reduced van der Waal interaction is the main factor that causes changes in lattice parameters. All systems exhibit semiconductor properties with indirect bandgap. The bandgaps of bulk, bilayer, and monolayer $\mathrm{SnSe}$ are $0.56 \mathrm{eV}, 0.84$ $\mathrm{eV}$, and $0.94 \mathrm{eV}$, respectively. Furthermore, the band structure of all systems shows a multi-valley band that can improve the thermoelectric properties. By reducing the dimensions from bulk to monolayer, the energy difference of the extrema I and II bands decreases until, in the monolayer system, we get $\Delta E_{12}$ of $0.05 \mathrm{eV}$. Under these conditions, we predict the monolayer SnSe will have the best thermoelectric properties compared to all systems. Our study demonstrates the novel electronic properties of the SnSe monolayer for flexible thermoelectric device applications.

\section{ACKNOWLEDGMENT}

This research was supported by "Program Riset Doktor untuk Sarjana Unggul (PMDSU)" research grant with contract number: 079/SP2H/LT/DRPM/2021.

\section{REFERENCES}

[1] L. Lin et al., Nitrogen cluster doping for highmobility/conductivity graphene films with millimeter-sized domains, Sci. Adv., 5, 8, 2019.

[2] G. H. Yang et al., Functionalization of Graphene and Applications of the Derivatives, J. Inorg. Organomet. Polym. Mater., 27, 1129-1141, 2017.

[3] E. B. Yutomo, F. A. Noor, and T. Winata, Effect of the number of nitrogen dopants on the electronic and magnetic properties of graphitic and pyridinic $\mathrm{N}$-doped graphene $-\mathrm{a}$ density-functional study, RSC Adv., 11, 18371-18380, 2021.

[4] E. B. Yutomo, F. A. Noor, and T. Winata, "Investigation of the Magnetic and Electronic Properties of Pyrrolic N-doped Graphene Using Density Functional Theory," J. Phys, Conf. Ser., 1951, 1, 2021.

[5] D. Sun et al., Ultrafast hot-carrier-dominated photocurrent in graphene, Nat. Nanotechnol., 7, 114-118, 2012.

[6] D. Li et al., Recent Progress of TwoDimensional Thermoelectric Materials, Nanomicro Lett., 12, 1, 2020.

[7] K. Kanahashi, J. Pu, and T. Takenobu, 2D Materials for Large-Area Flexible Thermoelectric Devices, Adv. Energy Mater., 10, 1-23, 2020.

[8] Y. Ye et al., Two-Dimensional GeSe as an isostructural and isoelectronic analogue of phosphorene: Sonication-assisted synthesis, chemical stability, and optical properties, Chem. Mater., 29, 8361-8368, 2017.

[9] M. F. Afsar, M. A. Rafiq, and A. I. Y. Tok, Two-dimensional SnS nanoflakes: synthesis and application to acetone and alcohol sensors, RSC Adv., 7, 21556-21566, 2017.

[10] T. V. Vu et al., Enhancement of monolayer SnSe light absorption by strain engineering: A DFT calculation, Chem. Phys., 521, 5-13, 2019.

[11] H. Q. Yang et al., Sn vacancy engineering for enhancing the thermoelectric performance of two-dimensional SnS, J. Mater. Chem. C, 7, 3351-3359, 2019.

[12] X. Lv et al., Two-dimensional GeSe for high performance thin-film solar cells, J. Mater. Chem. A, 6, 5032-5039, 2018.

[13] C. Liu et al., $\gamma$-GeSe: A two-dimensional ferroelectric material with doping-induced ferromagnetism, Appl. Phys. Lett., 115, 252904, 2019.

[14] L. Li et al., Single-Layer Single-Crystalline SnSe Nanosheets, J. Am. Chem. Soc., 135, 1213-1216, 2013.

[15] G. Ding et al., A comparative study of thermoelectric properties between bulk and monolayer SnSe, Results Phys., 15, 102631, 2019.

[16] F. Q. Wang et al., Thermoelectric properties of single-layered $\mathrm{SnSe}$ sheet, Nanoscale, 7 , 15962-15970, 2015.

[17] L. C. Zhang et al., Tinselenidene: a Twodimensional Auxetic Material with Ultralow Lattice Thermal Conductivity and Ultrahigh Hole Mobility, Sci. Rep. 6, 1-9, 2016. 
[18] L.-D. Zhao et al., Ultralow thermal conductivity and high thermoelectric figure of merit in SnSe crystals, Nature, 508, 373-377, 2014.

[19] S. Nag et al., Ultralow lattice thermal conductivity and anisotropic thermoelectric performance of AA stacked $\mathrm{SnSe}$ bilayer, Appl. Surf. Sci., 512, 145640, 2020.

[20] P. Giannozzi et al., Advanced capabilities for materials modelling with Quantum ESPRESSO, J. Phys. Condens. Matter, 29, 465901, 2017.

[21] P. Giannozzi et al., QUANTUM ESPRESSO: A modular and open-source software project for quantum simulations of materials, J. Phys. Condens. Matter, 21, 395502, 2009.

[22] P. E. Blöchl, Projector augmented-wave method, Phys. Rev. B, 50, 17953, 1994.

[23] J. P. Perdew, K. Burke, and M. Ernzerhof, Generalized gradient approximation made simple, Phys.Rev. Lett., 77, 3865-3868, 1996.

[24] C. G. Broyden, The convergence of a class of double-rank minimization algorithms 1 . General considerations, IMA J. Appl. Math., 6, 76-90, 1970.

[25] R. Fletcher, New approach to variable metric algorithms, Comput J., 13, 317-322, 1970.

[26] D. Goldfarb, A family of variable-metric methods derived by variational means, Math. Comput., 24, 23-23, 1970.

[27] D. F. Shanno, Conditioning of quasi-Newton methods for function minimization, Math. Comput., 24, 647-647, 1970.

[28] S. Grimme et al., A consistent and accurate ab initio parametrization of density functional dispersion correction (DFT-D) for the 94 elements H-Pu, J. Chem. Phys., 132, 154104, 2010.

[29] R. L. González-Romero, A. Antonelli, and J. J. Meléndez, Insights into the thermoelectric properties of $\mathrm{SnSe}$ from ab initio calculations, Phys. Chem. Chem. Phys., 19, 12804-12815, 2017.

[30] L. T. Yang et al., Influence of pressure on phase transition, electronic and thermoelectric properties of SnSe, J. Alloys Compd., 853, 157362, 2021.

[31] M. S. Gusmão et al., Thermoelectric properties of SnSe (Pnma) under hydrostatic pressure, Comput. Mater. Sci., 152, 243-247, 2018.

[32] Y. Suzuki and H. Nakamura, A supercell approach to the doping effect on the thermoelectric properties of SnSe, Phys. Chem. Chem. Phys., 17, 29647-29654, 2015.
[33] D. D. Cuong et al., Strain effect on electronic structure and thermoelectric properties of orthorhombic SnSe: A first principles study, AIP Adv., 5, 117147, 2015.

[34] H. Usui et al., Pudding-Mold-Type Band as an Origin of the Large Seebeck Coefficient Coexisting with Metallic Conductivity in Carrier-Doped $\mathrm{FeAs}_{2}$ and $\mathrm{PtSe}_{2}$, J. Electro. Mater., 43, 1656-1661, 2013.

[35] S. Wei et al., Strain tunable pudding-moldtype band structure and thermoelectric properties of $\mathrm{SnP}_{3}$ monolayer, J. Appl. Phys., 127, 155103, 2020.

[36] D. Qin et al., Strain-induced thermoelectric performance enhancement of monolayer $\mathrm{ZrSe}_{2}, R S C$ Adv., 7, 47243-47250, 2017.

[37] H. Y. Lv et al., Strain-induced enhancement in the thermoelectric performance of a $\mathrm{ZrS} 2$ monolayer, J. Mater. Chem. C, 4, 4538-4545, 2016.

[38] H. Y. Lv et al., Enhanced thermoelectric performance of phosphorene by straininduced band convergence, Phys. Rev. B, 90, $085433,2014$.

[39] Q. Wang et al., Strain Effect on Thermoelectric Performance of InSe Monolayer, Nanoscale Res. Lett., 14, 1-9, 2019. 